\title{
Lesion Sterilization Tissue Repair as Adjunct to Conventional Root Canal Treatment of Combined Periodontic-Endodontic Cases
}

\author{
${ }^{1}$ Ahmed A Saleh , ${ }^{2}$ Hossam A Eid, ${ }^{3}$ Khalid M Abdelaziz
}

\begin{abstract}
Background: Treatment of perio-endo lesions is challenging for the clinician. A combination of root canal treatment and periodontal treatment is needed in order to provide good results. There is a high failure rate due to the difficulty in eliminating the microflora that colonizes and complicates the lesion. Conventional root canal treatment (RCT) sometimes is not enough to prevent failure in such lesions. Hence the Aim of this study was to evaluate of use of LSTR (Lesion Sterilization Tisssue Repair) therapy in conjunction with, periodontal therapy and RCT in management of combined perio-endo lesions.
\end{abstract}

Materials and methods: This study was conducted on 26 patients with perio-endo lesions, The affected teeth were tender to percussion, presented no mobility, and meticulous periodontal probing revealed all pockets to be more than $6 \mathrm{~mm}$ in depth. Periapical radiographs of the teeth confirmed the extensive coronal carious lesion involving the pulp and revealed large periapical lesion. The patients were classified into two groups, each of 14 patients, control group (A) RCT done by using conventional sealer, and group (B), RCT done by mixing sealer with LSTR material in equal proportions. All teeth were treated by open flap debridement.

Results: In group B patients, after follow-up for 3, 6, 9 and 12 months, we found excellent prognosis (98\%), with no symptoms of pain and there was marked improvement in periodontal parameters for all teeth. However, on the other hand, in group A patients, the prognosis was found to be $90 \%$.

Conclusion: According to results of this study, we highly recommend the use of LSTR with sealer in RCT together with

\footnotetext{
${ }^{1,2}$ Assistant Professor, ${ }^{3}$ Associate Professor

${ }^{1}$ Department of Restorative Dental Sciences, College of Dentistry, King Khalid University, Abha, Saudi Arabia Department of Operative Dentistry, Faculty of Dentistry Almansoura University, Egypt

${ }^{2}$ Department of Preventive Dental Sciences, Division of Periodontics, College of Dentistry, King Khalid University, Abha Saudi Arabia; Department of Oral Medicine and Periodontology Faculty of Dentistry, Suez Canal University, Ismailia, Egypt

${ }^{3}$ Department of Restorative Dental Sciences, Division of Biomaterials, College of Dentistry, King Khalid University, Abha Saudi Arabia

Corresponding Author: Hossam A Eid, Assistant Professor Department of Preventive Dental Sciences, Division of Periodontics, Grager Campus, PO-3263, Abha-61471, Saudi Arabia Phone: 0530834985, e-mail: dentaleagle2011@gmail, hosam@ kku.edu.sa
}

periodontal treatment in management of perio-endo lesions to decrease the rate of failure and improve the periodontal condition.

Keywords: LSTR, RCT, Combined perio-endo lesion.

How to cite this article: Saleh AA, Eid HA, Abdelaziz KM. Lesion Sterilization Tissue Repair as Adjunct to Conventional Root Canal Treatment of Combined Periodontic-Endodontic Cases. World J Dent 2014;5(1):47-52.

Source of support: This work was financially supported by Deanship of Research, King Khalid University, Saudi Arabia. Project No. 119.

\section{Conflict of interest: None}

\section{INTRODUCTION}

The relationship between periodontal and pulpal disease was first described by Simring and Goldberg in $1964 .{ }^{1}$ Since then, the term, 'perioendo lesion' has been used to describe lesions due to inflammatory products found in varying degrees in both the periodontium and the pulpal tissues.

Removing the plaque biofilm and mineralized deposits from the tooth surface are fundamental aspects of periodontal therapy. However, completeness of periodontal debridement procedures decreases with increasing probing depth and furcation involvement. Locally administered adjunctive antimicrobials are difficult to maintain at a therapeutic concentration in the oral cavity and there is an increased concern regarding the development of antibiotic resistance. As a consequence, there is a need to develop alternative antimicrobial approaches for prophylactic and therapeutic periodontal regimes. $^{2,3}$

On the other hand, because of the complexity of the root canal system, complete elimination of endodontic infection cannot always be achieved. Bacteria located in isthmuses, ramifications, dentinal tubules and irregularities of the root canal walls may be unaffected by endodontic disinfection procedures. Bacterial antigens egressing into the periapical area maintain the periapical inflammation and ultimately cause endodontic treatment failures. ${ }^{4,5}$

Lesion sterilization and tissue repair therapy (LSTR) is also called NIET or noninstrumental endodontic treatment as it claims to be 'a new biologic approach in the treatment 
of carious lesions with or without pulpal and periapical involvement using a mixture of 3 antibiotics.' This concept was developed by the Cariology Research Unit of the Niigata University School of Dentistry in $1988 .{ }^{6}$

LSTR technique involves the use of three broad spectrum antibiotics namely, Metronidazole, Ciprofloxacin and Minocycline. There are conflicting data on the ratio and proportion mixture of the antibiotics. Some proponents endorse a 3:1:1 mix, Garcia et al endorses 3:1:3 mix. At present Hoshino is endorsing a 1:1:1 mix. The rationale behind the change in the ratio of the mix is unknown. The three antibiotics are mixed with propylene glycol or macrogol, 'which is found to be an excellent vehicle to carry 3 Mix into the entire dentin and through the dentinal tubules and kill all the bacteria in lesions. ${ }^{7}$

LSTR 3Mix is said to be 'bactericidal to aerobic bacteria and to resistant obligate anaerobes' Hoshino et al identifies the anaerobic microorganisms recovered from carious dentin: $80 \%$ are obligate anaerobes; from infected pulp: $92 \%$ obligate anaerobes; from infected root canal dentin: $80 \%$ obligate anaerobes. Obligate anaerobes are sensitive to metronidazole that's why it is the first choice because of its wide bactericidal spectrum against anaerobes. However, since all bacteria cannot be eliminated by metronidazole alone, other drugs may be necessary to sterilize the infected dentin. They have added ciprofloxacin and minocycline. In the absence of Minocycline in the market, cephalexin was substituted. ${ }^{5,6}$

The Aseer region (population of 1,200,000) is located in the southwest of Saudi Arabia covering an area of more than $80,000 \mathrm{~km}^{2}$. The region extends from the high mountains of Sarawat (with an altitude of 3,200 meters above the sea level) to the Red Sea, and lies few kilometers from the northern border of the neighboring Yemen. Dental services in the region are provided through a wide spread network of primary healthcare centers and a tertiary services provided by the College of Dentistry, King Khalid University (KKU).

This study is an attempt to provide a rational approach to the perio-endo/endoperio question based on a review of the relevant literature. In the light of evidence, clinical concepts for the diagnosis and treatment of lesions involving both periodontal and pulpal tissues.

\section{MATERIALS AND METHODS}

\section{Patients and Teeth involved in This Study}

Each of the patients involved in the study. Prior to their inclusion to this study, they were informed of the aim, expected clinical results, as well as the risks and alternatives of the treatment, and they subsequently completed an approved informed consent.
The affected teeth were tender to percussion, presented no mobility and meticulous periodontal probing measurements revealed all pockets to be more than $6 \mathrm{~mm}$ in depth. A periapical radiograph of the teeth confirmed the extensive coronal carious lesion involving the pulp, periodontal lesions either showed a widening of the periodontal ligament space in the periapical area with an intrabony defect, deep periodontal pockets, and or furcation involvement as well as revealed a large periapical lesion.

The patients of this study were classified into two groups. group A (control group) consisted of 12 patients with perioendo lesions who were treated by open periodontal flap debridement, conventional root canal treatment (RCT) and obturated with conventional sealer.

On the other hand, Group B consisted of those patients who were treated by open periodontal flap debridement and conventional root canal treatment (RCT), obturation with conventional sealer mixed with LSTR 3 mix in equal proportions. Final restoration for all the concerned teeth in both groups were done with glass ionomer cement (Ketac TM Cem Aplicap, Seefeld, Germany).

\section{Preparation of the Three Mix}

For the Ciprofloxacin tablet, the enteric coating was removed using a scalpel. The tablet was pulverized using mortar and pestle. For the Metronidazole and Minocycline which are available in capsules, the powder was segregated. Antibiotics were stored and sealed in air tight containers. The three antibiotics were mixed together with propylene glycol and a creamy consistency was prepared.

\section{Root Canal Treatment}

After rubber dam isolation and removal of all carious tissue and access cavity preparation, a working length was taken without local anesthesia due to pulp necrosis during canal preparation, abundant irrigation with $2.5 \%$ sodium hypochlorite was carried out after each instrument. The canals were dried and filled with calcium hydroxide powder mixed with sterile saline. This procedure was repeated till the canals were completely dry and there was no evidence of odor or discharge. The canals were then filled with lateral condensation technique using conventional sealer in group A and sealer mixed with 3 mix LSTR in group B. Finally all teeth were restored with glass ionomer cement.

\section{Periodontal Management}

After the first endodontic visit, sign and symptoms of pain and swelling were relieved. Periodontal probing depths were measured by William graduated probe before the flap procedure as well as periodontal attachment loss and bone 
loss for comparison with these measurement after 3, 6, 9 and 12 months follow-up period. Periapical radiographs were used from the preoperative visit and throughout the whole period of study at an interval of 3, 6, 9 and 12 months respectively.

It was decided to manage the periodontal defect for all teeth in both groups after 1 month after the endodontic therapy. On the day of the surgery, the surgical site was anesthetized by buccal and palatal/lingual infiltrations of 2\% lignocaine with 1:80,000 adrenalin local anesthetic solutions. Buccal mucoperiosteal flaps were raised by intrasulcular incisions from the mesial aspect of affected tooth to the mesial aspect of adjacent tooth to remove the local factors of microbial plaque biofilm, calculus and diseased root cementum as well as dentin. The flap was replaced and firm pressure applied using a moist gauze pack to minimize blood clot formation beneath the flap and allow better approximation of the flap to the bone. Four sutures (4/0) were placed to secure the flap. Firm pressure was reapplied and postoperative instructions given. One week after the surgery, the patient returned asymptomatic for suture removal.

\section{RESULTS}

Twelve-month clinical and radiographic follow-ups were carried out on 24 patients of both groups divided into 3, 6, 9 and 12 months intervals respectively. Two patients each from both the groups were lost for follow-up resulting in total of 22 patients.

Pain with was complained in three teeth in group A patients in the first month and in one tooth in group $B$ in the third month. However, symptoms markedly reduced in the following months for both groups.

During the follow-up periods, two teeth exhibited clinical and radiographic failure from the control group A and no teeth exhibited clinical and radiographic failure in study group $\mathrm{B}$.

At all the selected sites, the following clinical parameters, probing depth, clinical attachment level, gingival margin location, defect depth were assessed at baseline, 6, 9 and 12 months postsurgery using William graduated periodontal probe and defect depth by means of an intraoral periapical radiograph, there was significant improvement in these parameters in the study group B than in group $\mathrm{A}$. The pre and post-periosurgery differences in all periodontal parameters are depicted in Tables 1 and 2 respectively.

In study group B: At baseline, the probing depth ranged from 5 to $10 \mathrm{~mm}$, clinical attachment level 3 to $10 \mathrm{~mm}$, gingival margin location -2 to $2 \mathrm{~mm}$ and defect depth 1.4 to $11.8 \mathrm{~mm}$ respectively. At 9 months postperiodontal surgery, the probing depth ranged from 2 to $6 \mathrm{~mm}$, clinical attachment level 2 to $7 \mathrm{~mm}$, gingival margin location -3 to $0 \mathrm{~mm}$ and the defect depth 1.6 to $6.3 \mathrm{~mm}$. The mean pocket depth at baseline was $7.25 \pm 2.05$ which reduced to $3.63 \pm 1.41,9$ months postsurgery. The mean reduction in pocket depth when compared from baseline to 9 months was $3.62 \pm 1.19$ which was statistically significant. The mean clinical attachment level at baseline was $6.00 \pm 2.73$ whereas values after 9 months postsurgery was $4.13 \pm 2.19$. The mean gain in clinical attachment level when compared from baseline to 12 months postsurgery was $-0.50 \pm 1.20$. The mean change in gingival margin location when compared from baseline to 9 months was $1.75 \pm 0.71$ which was significant.

In group A: At baseline the probing depth ranged from 6 to $11 \mathrm{~mm}$, clinical attachment level 3 to $12 \mathrm{~mm}$, gingival margin portion -2 to $1 \mathrm{~mm}$, defect depth 2.4 to $15.6 \mathrm{~mm}$. After 9 months postsurgery, the probing depth ranged from 2 to $5 \mathrm{~mm}$, clinical attachment level 1 to $7 \mathrm{~mm}$, gingival margin position -4 to $0 \mathrm{~mm}$ and the defect depth 0 to $11.8 \mathrm{~mm}$. The mean pocket depth at baseline was $10.38 \pm$ 3.16 which reduced to $3.88 \pm 0.99$ after 9 months postsurgery. The mean reduction in pocket depth when compared from baseline to 9 months was $6.50 \pm 2.51$. The mean clinical attachment level at baseline was $9.25 \pm 3.20$ whereas values after 9 months postsurgery was $4.63 \pm 2.07$. The mean gain in clinical attachment level when compared from baseline to 9 months was $4.62 \pm 2.26$. The mean gingival margin position at baseline was $1.18 \pm 1.36$ whereas values after 9 months postsurgery was $-0.75 \pm 1.83$. The mean change in gingival margin position when compared from baseline to 9 months was $1.88 \pm 1.25$.

\section{Radiographic Changes}

In study group $B$, the mean baseline defect depth was $6.41 \pm 3.75$ whereas that after 9 months postsurgery was $3.19 \pm 1.84$. The mean difference from baseline to 9 months was 2.65 and the mean percentage of original defect fill at 9 months postsurgery was $31.2 \pm 61.4$. On the other hand, the mean baseline defect depth in Group A was $9.59 \pm 4.95$ whereas that after 12 months postsurgery was $6.63 \pm 4.66$. The mean difference from the baseline to 9 months was 3.74 and mean percentage fill of original defect at 9 months post-surgery was $40.7 \pm 31.7$ respectively. The preoperative and postoperative radiographs are shown in Figures 1A to $\mathrm{C}$ and Figures 2A to $\mathrm{C}$ respectively.

\section{DISCUSSION}

The pulp and periodontium have embryonic, anatomic and functional interrelationships. Embryonic development gives rise to anatomical connections which remain throughout the life of the tooth. ${ }^{8}$ The apical foramen decreases in size as the 


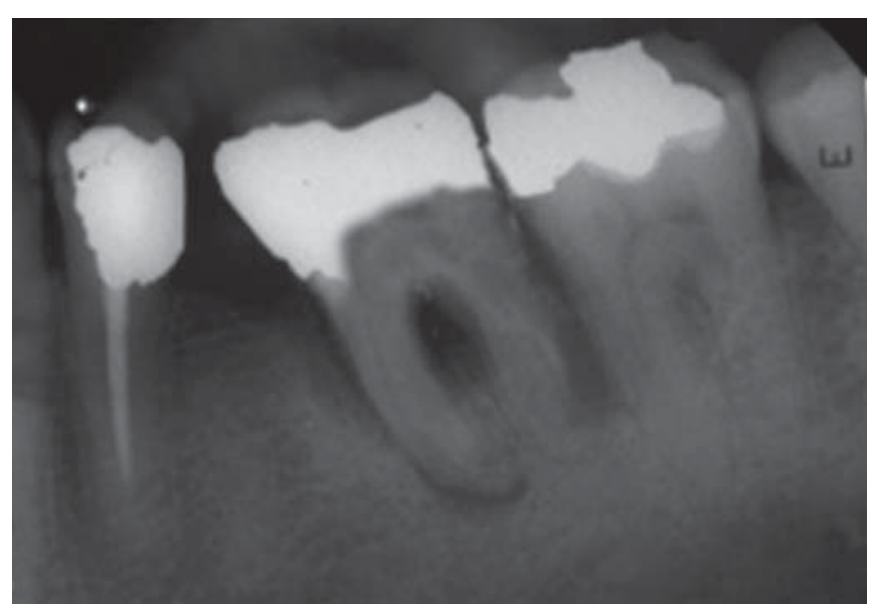

Fig. 1A: Preoperative X-ray

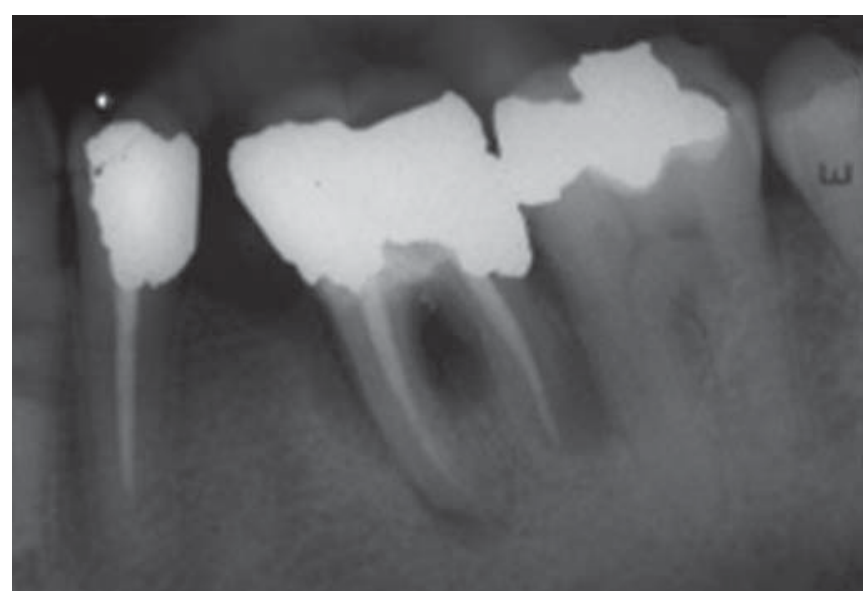

Fig. 1B: After 9 months

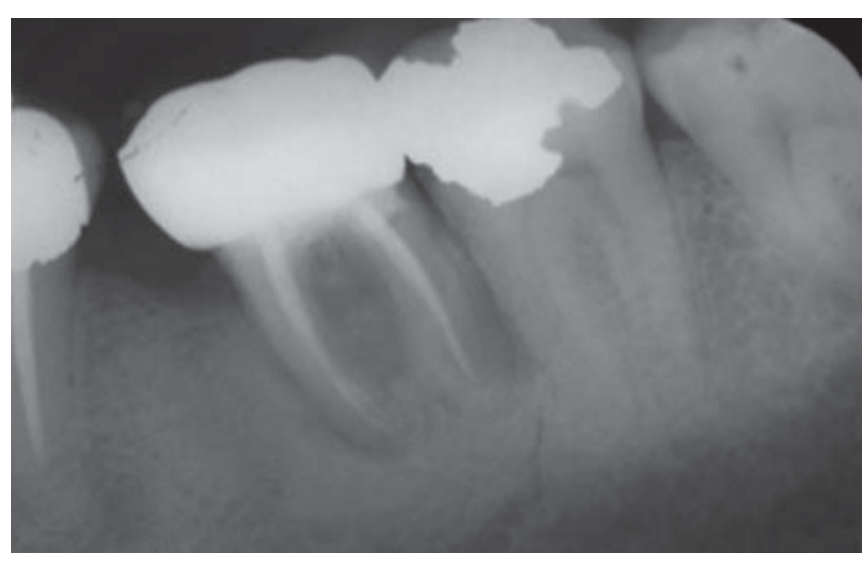

Fig. 1C: After 12 months

proliferation of the Sheath of Hertwig continues. It remains patent and serves as the communication on which the pulpal tissues rely for nutrition and nervous innervation. As the root develops, ectomesenchymal channels get incorporated, either due to dentin formation around existing blood vessels, or breaks in the continuity of the Sheath of Hertwig, to become accessory or lateral canals. The majority of accessory canals

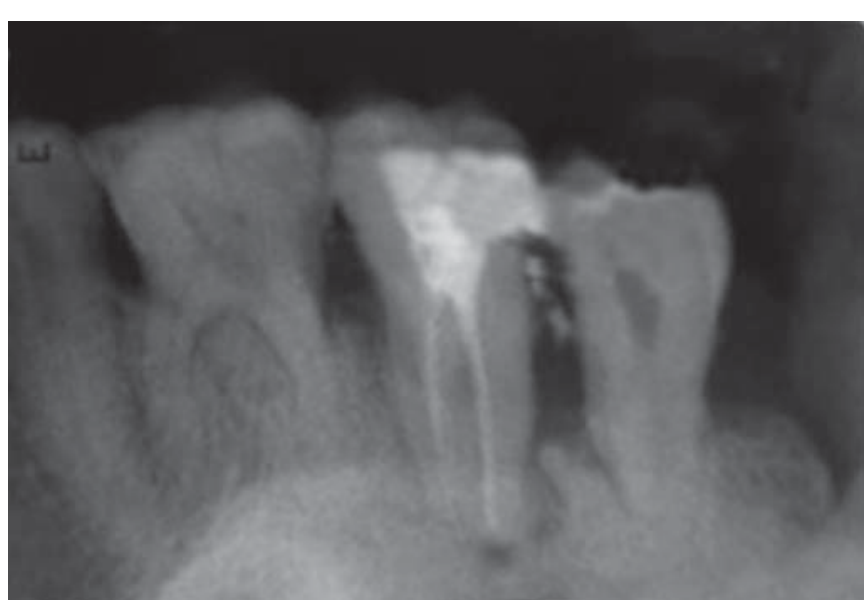

Fig. 2A: Immediately after obturation

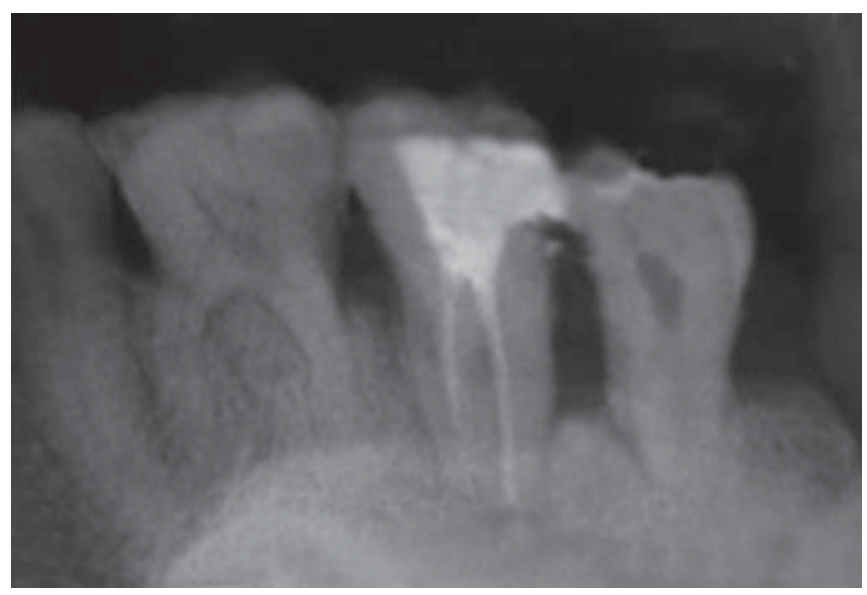

Fig. 2B: After 9 months

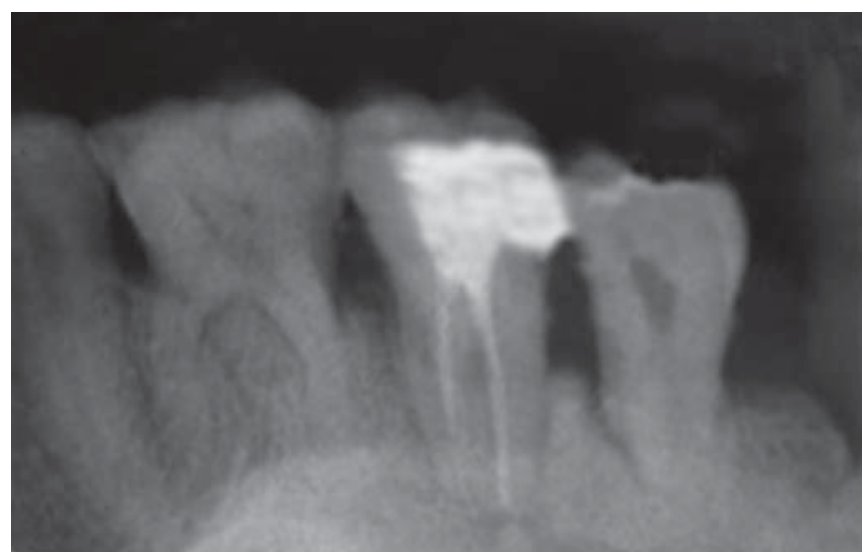

Fig. 2C: After 12 months

are found in the apical part of the root and lateral canals in the molar furcation regions. Tubular communication between the pulp and periodontium may occur when dentinal tubules become exposed to the periodontium due to the absence of overlying cementum. These are the pathways that may provide the means by which pathological agents pass between the pulp and periodontium, thereby creating the perioendo lesion. .,10 $^{9}$ 
Table 1: Control group I (mean values)

\begin{tabular}{llllll}
\hline & Baseline & 3 months & 6 months & 9 months & 12 months \\
\hline PPD & $10.38 \pm 3.16 \mathrm{~mm}$ & $8.44 \pm 4.21 \mathrm{~mm}$ & $6.66 \pm 31 \mathrm{~mm}$ & $5 \pm 5.78 \mathrm{~mm}$ & $4.88 \pm 0.99 \mathrm{~mm}$ \\
CAL & $9.25 \pm 3.20 \mathrm{~mm}$ & $8 \pm 8.32 \mathrm{~mm}$ & $5.8 \pm 4.34 \mathrm{~mm}$ & $4.95 \pm 1.16 \mathrm{~mm}$ & $4.63 \pm 2.07 \mathrm{~mm}$ \\
GML & $1.18 \pm 1.36 \mathrm{~mm}$ & $1.05 \pm 0.88 \mathrm{~mm}$ & $0.88 \pm 0.66 \mathrm{~mm}$ & $-0.5 \pm 0.35 \mathrm{~mm}$ & $-0.75 \pm 0.83 \mathrm{~mm}$ \\
BDD & $6.41 \pm 3.75 \mathrm{~mm}$ & $15 \pm 2.77 \mathrm{~mm}$ & $22.45 \pm 2.86 \mathrm{~mm}$ & $26.12 \pm 1.76 \mathrm{~mm}$ & $29.2 \pm 2.4 \mathrm{~mm}$
\end{tabular}

$\mathrm{BD}(\%)$

PPD: periodontal pocket depth; CAL: clinical attachment level; GML: gingival margin location; BDD: bone defect depth; $\mathrm{BD}$ : bone density

Table 2: Study group II (mean values)

\begin{tabular}{llllll}
\hline & Baseline & 3 months & 6 months & 9 months & 12 months \\
\hline PPD & $7.25 \pm 2.05 \mathrm{~mm}$ & $6.34 \pm 2.3 \mathrm{~mm}$ & $4 \pm 1.1 \mathrm{~mm}$ & $3.55 \pm 1.22 \mathrm{~mm}$ & $3.62 \pm 1.19 \mathrm{~mm}$ \\
CAL & $6 \pm 2.73 \mathrm{~mm}$ & $5.4 \pm 2.1 \mathrm{~mm}$ & $5 \pm 2.6 \mathrm{~mm}$ & $4.88 \pm 2.12 \mathrm{~mm}$ & $4.13 \pm 2.19 \mathrm{~mm}$ \\
GML & $1.18 \pm 1.36 \mathrm{~mm}$ & $0.92 \pm 0.11 \mathrm{~mm}$ & $0.73 \pm 0.61 \mathrm{~mm}$ & $-0.9 \pm 0.15 \mathrm{~mm}$ & $-0.25 \pm 0.03 \mathrm{~mm}$ \\
BDD & $8.23 \pm 2.66 \mathrm{~mm}$ & $18 \pm 2.45 \mathrm{~mm}$ & $24.06 \pm 1.44 \mathrm{~mm}$ & $29.22 \pm 1.33 \mathrm{~mm}$ & $38.8 \pm 2.66 \mathrm{~mm}$ \\
BD(\%) & & & & & \\
\hline
\end{tabular}

PPD: periodontal pocket depth; CAL: clinical attachment level; GML: gingival margin location; BDD: bone defect depth; $\mathrm{BD}$ : bone density

The responses of the pulp to traumatic injury to the periodontal membrane (PDM) require special consideration, particularly with respect to the assessment of pulp vitality, and the determination of cases requiring pulp extirpation in order to avoid inflammatory root resorption. Although the pulp is relatively isolated from the rest of the dentoalveolar complex by a dentin/cementum barrier, it is important to remember that it can communicate with the PDM through apical and lateral foramina, and areas of damaged cementum. Hence, it is a priority to both preserve the integrity of the cemental layer in cases of traumatic injury and periodontal disease, and to prevent the inflammation and resorption associated with periapical lesions by accurate diagnosis of irreversible pulpitis and pulp necrosis, followed by appropriate endodontic debridement procedures. ${ }^{11}$

Intrabony defects associated with deep periodontal pockets, are ecological niches for periodontal pathogens, and if left untreated, they complicate the outcome of any therapy performed on the tooth. In most cases of endoperio lesions, clinical symptoms disappear following successful endodontic therapy. However, it becomes essential to correct the periodontal defect simultaneously in these cases to prevent recurrence, and to improve the functional status of the tooth. ${ }^{12}$

It has been reported that certain microorganisms may survive outside the root canal and maintain the inflammatory disease in periapical tissues. This has been suggested to happen either by microbes adhering to the apical root surface, forming biofilm-like structures, ${ }^{13}$ or by their existing within the inflamed periapical tissues, usually as cohesive colonies, becoming independent of the root canal infection. Bacteria of the genera Actinomyces and Arachnia may prevent periapical healing in this way by establishing themselves in the periapical tissues. ${ }^{14}$ The presence of bacteria on the root surface in failed endodontic treatment has also been reported in histomorphological studies ${ }^{15}$ Perio-endo lesions are common conditions that are often difficult to diagnose and persistent if not treated completely. However, if the patient's history is taken carefully and thorough evaluation of all possible routes of infection is carried out, these lesions can be completely eliminated to give excellent results. A correct diagnosis helps in formulating a correct treatment plan and in most of the cases, a properly done endodontic treatment is sufficient to eliminate the infection. Wherever a secondary periodontal involvement exists, it requires specific therapy to achieve success. Most of these lesions have a vertical osseous defect and regenerative therapy gives excellent results after open flap debridement. ${ }^{16}$ The discharge of pus from the periodontal pocket of a tooth had persisted despite the root canal retreatment. This may have been a result of persistent periodontal disease, intracanal microorganisms that were inaccessible to instrumentation (Sundqvist et al 1998) or coronal leakage (Ray \& Trope, 1995). However, the LSTR hypothesis proposes that the local application of antimicrobial agents, such as the 3Mix-MP combination evaluated in the present study, would disinfect these lesions by eradicating bacteria in dentinal or pulpal lesions. The results in the present clinical study support this concept since $>90 \%$ of cases fulfilling the criteria of pain free and absence of tenderness having a good long-term outcome after local application of 3Mix-MP. ${ }^{17,18}$

The LSTR hypothesis predicts a successful clinical outcome by local bacterial eradication. According to Hoshino, $94 \%$ of the cases that experienced pain were relieved within 
24 hours after LSTR 3 Mix, regardless of the nature and severity of pain. ${ }^{7}$ In addition to the treatment of pulpitis cases that were reported in the present study, permanent teeth $^{18-20}$ and primary teeth ${ }^{19,21-23}$ with periradicular lesions have been also successfully treated by 3Mix-MP endodontic therapy. These might indicate that 3Mix-MP therapy may have broad applications for endodontic treatment by creating conditions for pulpal healing, revascularization or control of infection. For example, it is also possible to provide revisionary endodontic treatment (i.e. 'retreatment') of cases with 3Mix-MP without removal of previous root canal obturating materials (Takushige and Hoshino, 2004). The results of this clinical study indicate that LSTR therapy using 3Mix-MP, a mixture of metronidazole, ciprofloxacin and minocycline (3Mix), and macrogol and propylene glycol (MP), provided excellent clinical outcomes in treatment of combined perioendo lesions together with periodontal therapy and conventional root canal treatment to decrease the failure possibility.

\section{CONCLUSION}

According to the results of this study successful treatment can be attributed to a correct diagnosis, successful endodontic therapy with the use of LSTR as a sealer in conjunction with periodontal therapy in management of combined perio-endo lesions.

\section{ACKNOWLEDGMENT}

This work was financially supported by Second Research Project Grant from Deanship of Research, King Khalid University (KKU), Abha city, Kingdom of Saudi Arabia, Project No. (KKU_S119_33). Authors acknowledged Dr Amar Shulaporkar, Assistant Professor, Oral Medicine, for his valuable effort in editing this work.

\section{REFERENCES}

1. Simring M, Goldberg M. The pulpal pocket approach: Retrograde periodontitis. J Periodontol 1964;35:22-48.

2. Dai T, Huang Y, Hamblin MR. Photodiagnosis and Photodynamic Therapy. Sep-Dec 2009;6(3-4):170-188.

3. Carranza FA. The role of calculus in the etiology of periodontal disease. In: Clickman's Clinical Periodontology, 7th ed. Philadelphia: WB Saunders, pp. (1990); 394-395.

4. Siqueira JF Jr. Aetiology of root canal treatment failure: why well-treated teeth can fail. International Endodontic Journal 2000;34:1-10.

5. Lin LM, Skribner JE, Gaengler P. Factors associated with endodontic treatment failures. Journal of Endodontics 1992;18: 625-627.
6. Guidelines on Appropriate Use of Antibiotics for Pediatric Patients, Council on Clinical Guidelines, 2003.

7. Garcia C, Cruz E, Hoshino E, Nunez P. 3Mix Lesion Sterilization and Tissue Repair Therapy (LSTR) in Extensive Caries of Deciduous Teeth. The Journal of the Philippine Dental Association 2004;56(1):4-13.

8. Ten Cate AR. Oral histology-development, structure and function. 4 th ed. Mosby; 1994.

9. Christie WH, Holthuis AF. The endo-perio problem in dental practice: Diagnosis and prognosis. JADA 1990;56:1005-1011.

10. Whyman RA. Endodontic-periodontic lesions. Part 1; prevalence, aetiology and diagnosis. New Zealand Dent J 1988;84:74-77.

11. Solomon C, Chalfin H, Kellert M, Weseley P. The endodonticperiodontal lesion: a rational approach to treatment. J Am Dent Assoc 1995;126:473-479.

12. Pierce A, Pulpal injury: pathology, diagnosis and periodontal reactions. Aust Endodontic J 1998 August;24(2):60-65.

13. Takushige T, Cruz EV, Moral AA, Hoshino E. Endodontic treatment of primary teeth using a combination of antibacterial drugs. International Endodontic Journal 2004;37:132-139.

14. Zehnder M, Gold SI, Hasselgren G. Pathologic interactions in pulpal and periodontal tissues Columbia University, School of Dental and Oral Surgery, Division of Endodontics, New York, NY 10032, USA. Clin Periodontology 2002 August 29;(8): 663-671.

15. Tronstad L, Barnett F, Cervone F. Periapical bacterial plaque in teeth refractory to endodontic treatment. Endodontics and Dental Traumatology 1990;6:73-77.

16. Happonen RP. Periapical actinomycosis: a follow-up study of 16 surgically treated cases. Endodontics and Dental Traumatology 1986;2:205-209.

17. Sjögren U, Happonen RP, Kahnberg KE, Sundqvist G. Survival of Arachnia propionica in periapical tissue. International Endodontic Journal 1988;21:277-282.

18. Figdor D, Sjögren U, Sörlin S, Sundqvist G, Nair PNR. Pathogenicity of Actinomyces israelii and Arachnia propionica: experimental infection in guinea pigs and phagocytosis and intracellular killing by human polymorphonuclear leukocytes in vitro. Oral Microbiology and Immunology 1992;7:129-136.

19. Bergenholtz G, Lekholm U, Liljenberg B, Lindhe J. Morphometric analysis of chronic inflammatory periapical lesions in root-filled teeth. Oral Surgery Oral Medicine Oral Pathology 1983;55: 295-301.

20. Shenoy N, Shenoy A. Endo-perio lesions: diagnosis and clinical considerations. Indian J Dent Res [serial online] 2010 [cited 2011 Apr 10];21:579-585.

21. Rotstein I, Simon JH. Diagnosis, prognosis and decision-making in the treatment of combined periodontal-endodontic lesions. Periodontol 2000, 2004;34:165-203.

22. Karabucak B, Setzer FC. Conventional and surgical retreatment of complex periradicular lesions with periodontal involvement. J Endod 2009 Sep;35(9):1310-1315. Epub 2009 Jul.

23. Sculean A, Nikolidakis D, Schwarz F. Regeneration of periodontal tissues: combinations of barrier membranes and grafting materials-biological foundation and preclinical evidence: a systematic review. J Clin Periodontol 2008 Sep;35(8 Suppl):106-116. 